\title{
Folie et création romanesque en Afrique ${ }^{1}$ Alexie Tcheuyap Queen's University (Kingston, Ontario)
}

D epuis la publication de L'État sauvage et Les Flamboyants, romans assimilant de manière simplificatrice le Nègre à la folie, on observe, avec un peu de recul, que cette thématique inspire de plus en plus les romanciers en Afrique. En présentant l'Africain sous la catégorie de la maladie, on pourrait être tenté, au premier abord, de penser qu'ils ont définitivement succombé à ce que Bernard Mouralis désigne "I'écran séducteur". Autrement dit, ils auraient intégré le discours travestissant, à la limite raciste, de certains amateurs de littérature exotique. Une telle conclusion serait rapide, car il existe un discours africain sur la folie que la présente étude voudrait élucider, mieux cerner. Une telle problématique pourrait permettre de recentrer le débat sur la question, réactualiser les hypothèses, et reconsidérer l'être au monde du Nègre, son identité rendue par l'anormal. Mais qu'est-ce que la folie ? Et la folie en Afrique?

On pourrait se poser mille fois cette question, car la notion est éternellement controversée et même dangereuse. En considérant les oeuvres de Mongo Beti, Mariama Bâ, Tahar Ben Jelloum, Birago Diop, Pius Ngandu Nkashama ou Sony Labou Tansi, on observe simplement que la déraison y occupe une place centrale. La manière de la traiter varie aussi considérablement. Ils reprennent la notion qui, dans leurs romans devient lancinante, presque obsédante. On aurait pu penser que, longtemps après que Frantz Fanon ait révélé le rapport entre guerres coloniales et troubles mentaux, le Nègre aurait recouvré son équilibre psychique. Mais les auteurs convoqués semblent rappeler plutôt le contraire. Pourquoi demeure-t-il donc caractérisé par l'anormal? En quoi la démence continue-t-elle d'informer sa condition? Peut-on, en vertu des nouvelles trajectoires esthétiques observées, déterminer une poétique de la folie dans le roman africain? 
Telles sont les principales interrogations à élucider. Sans invalider ou nier la théorie anthropologique d'Ibrahima Sow ayant informé l'étude d'Éliane Saint André Utudjian, on tentera de la dépasser pour considérer la démence dans toute sa complexité: anormalité, lecture sociale du comportement, dissidence (Ambroise Kom), clinique, psychiatrique et psychanalytique (Pius Ngandu Nkashama, Alexie Tcheuyap), mais surtout idéologique, philosophique et politique (Fanon, Michel Foucault). L'hypothèse est que l'Africain étant devenu moins un sujet culturel ou religieux que citoyen d'un État chaotique, il a de nouvelles relations au monde. En admettant que toute identité est moulée par les conditions idéologiques et objectives de l'instant, on comprend que les hères déchues des textes de Ngandu Nkashama ne sombrent pas dans le délire de la même manière que Sarzan, le militaire prétentieux et vaniteux des Contes d'Amadou Koumba. On comprend également que Moha de Ben Jelloum, exclu par un consensus social déviant, n'est peut-être pas aussi malade que les figures tragiques peuplant le roman et qui, en réalité, méritent un internement bien contrôlé. On l'a probablement perçu, la notion de folie est donc extrêmement dynamique et cela relève surtout de la diversité des causes qu'on lui attribue. Elles semblent varier avec le sexe.

\section{Les Traumatismes masculins}

Utudjian et Sow l'ont illustré, il est possible d'aborder la question de la déraison en Afrique en analysant les rapports entre le sujet malade et sa communauté. L'argument majeur de Sow réside dans la nécessité de constituer un schéma théorique tenant compte des horizons anthropologiques dans la causation de la folie. Or comme le soutient ma récente étude (2), l'Afrique évolue dans un cadre où sévissent de nouvelles données tragiques. En effet, "Il y a une sorte d'irréductibilité de la folie que la prise en compte du milieu socio-culturel ne suffit pas à élucider complètement" (Mouralis 11). Le roman négro africain en arrive donc de plus en plus à témoigner contre l'hypothèse anthropologique et culturelle. Pourtant, une réconciliation est possible entre les bases épistémologiques de Sow et la catégorie psycahanalytique qu'il disqualifie. C'est notamment le cas en ce qui concerne les notions de conflits et de traumatisme qu'on peut observer dans "Sarzan" de Birago Diop.

Du fait d'un épisode dans l'armée coloniale, trempé de quelques aspects du matérialisme et du rationnlisme occidentaux lui ayant lavé le cerveau, Sarzan retourne dans son village et se signale par la profanation des symboles d'une tradition l'ayant pourtant nourri. Pour fertiliser les champs, il estime idiot de procéder au sacrifice des poulets noirs dont on asperge le sang à certains coins, recommandant le travail. Il interrompt la cérémonie rituelle de la circoncision et de l'excision. Il décroche et jette loin de sa case le sac contenant les génies protecteurs de sa famille. Il se rend dans le bois sacré et y détruit tous "fétiches". Il coupe et brûle les branches de l'arbre sacré. De telles offenses, doublées de qualificatifs péjoratifs comme "manières de sauvages", "superstition", ne peuvent rester 
impunis. Jaloux, les Dieux ne tardent pas à réagir à l'opprobre lancé contre leur existence. Sarzan qui, par une autre bravade s'installe sous l'arbre sacré, est précipité dans le délire:

Il parlait lorsque soudain, il sentit comme une piqûre à son épaule gauche; il se retourna. Quand il regarda à nouveau ses auditeurs, ses yeux n'étaient plus les mêmes. Une bave mousseuse et blanche naissait aux coins de ses lèvres. Il parla, et ce n'étaient pas les mêmes paroles qui sortaient de sa bouche. Les souffles avaient pris son esprit et ils criaient maintenant leur crainte. (185)

La démence subite qui s'empare de Sarzan est une preuve de la nature vengeresse et conservatrice des Dieux africains. Un esprit vengeur s'abat sur le psychisme de Sarzan qui, en perdant son nom Thiemoko Keita, perd ainsi son identité. On demeure là dans la logique du conflit entre le sujet et sa communauté entière, dont sa famille. La transgression de l'ordre culturel fait déployer le chaos social. Le divorce étant consommé entre le macrocosmos, le mésocosmos et le microcosmos (Sow), l'auteur de la transgression sombre dans le délire. Mais l'hypothèse du traumatisme psychique peut être aussi recevable dans ce cas.

En effet, le traumatisme psychique, comme cause immédiate et événénement déclenchant, part d'un moment et d'un fait pathogènes. Le cas de Sarzan le montre bien, en dépit du fait qu'il soit consécutif à un conflit durable et insoutenable, c'est à un moment précis, inattendu et violent que le sujet bascule dans le délire. Et Birago Dop rend bien cette soudaineté par l'usage du passé simple. L'installation de Sarzan sous l'arbre sacré, acte supprême de transgression, constitue le déclic précipitant le sujet dans le délire. Son ultime bravade se révèle donc irréversiblement traumatogène et peut valider une lecture psychalytique, du moins au niveau de l'interprétation. On peut en dire autant de L'Histoire du fou

C'est également dans des circonstances traumatiques que Zoaétoa, dans $L$ 'Histoire $d u$ fou, est précipité à la rupture psychique. Poussé à l'extrême par sa mégalomanie et la recherche frénétique de l'accumulation, il fréquente les cercles du pouvoir jusqu'à en devenir l'un des bras séculiers. Membre de l'un des nombreux organes étatiques de répression, en mission commandée, il assassine sans le savoir son propre frère pour lequel il nourrissait du reste une profonde hostilité. "Depuis que Zoaétoa connaît la nature et l'étendue du crime, son âme trop fragile n'a pas pu survivre" (211). Connaître étant une tragédie comme le formulait Nietzsche, il devient fou et erre dans les rues, offrant en spectacle toute la nudité de son anatomie. On le voit dans ces deux cas, si les cadres anthropologiques sont différents, on peut relever des événéments déclenchants de même nature. Aussi bien les conflits socio-culturels que les dérives de la société civile provoquent à des moments précis une rupture fondamentale dans la personnalité. Du fait de 
traumatismes nés de situations inattendues, le sujet bascule dans la démence. Dans Le Pacte de sang, Vieux Hizambe connaît exactement la même trajectoire clinique. La découverte d'une tête sous un lit, avec des yeux clignants, provoque aussitôt un choc et il dérape dans le délire. Comme Zoétoa, son drame vient de la connaissance, de la vérité. Les fétiches de sa concubine que sa curiosité l'amène à découvrir le précipite aussitôt dans la catastrophe. Mais si la surprise traumatique détraque les hommes, avec les femmes, la folie est plutôt le résultat de durables frustrations.

\section{Les Frustrations féminines}

Dans Un Chant écarlate, Mariama Bâ retrace l'itinéraire de Mireille, jeune française aux ambitions généreuses, contrainte de "[faire] durement deux apprentissages :celui de la vie conjugale et celui de femme de Nègre en Afrique "(151). Son cas a des origines d'abord culturelles, car elle ne parvient pas à intégrer définitivement les modes de la vie familiale en Afrique, modes décrits avec un superlativisme précis par un narrateur sournoisement féminin.

En effet, elle ne tolère pas les visites trop fréquentes de sa belle-mère, par ailleurs suffisamment hostile, ni celle des amis de son mari qui se prélassent bruyamment dans son salon et diminuent sa ration alimentaire. En plus de ce qu'elle considère comme une " agression quotidienne de son intimité", les excentricités de son époux aux instincts dominateurs, et dont le manque d'hygiène élémentaire l'agacent, achèvent de malmener son existence. Mais c'est l'infidélité d'Ousmane qui porte un coup fatal à son psychisme. Après lui avoir fait subir des assauts sexuels inspirés par une amante, il déserte régulièrement le domicile conjugal pour se rendre chez celle-ci. Il l'épouse clandestinement, au plus grand bonheur de sa famille.

Réalisant l'ampleur de la félonie, torturée par le désir et la solitude, Mireille redécouvre les lettres d'amour de leur belle époque d'idylle. La beauté et la candeur des termes contredit le drame hallucinant dans lequel elle se débat. Les lettres ont un effet traumatique évident car un délire s'empare immédiatement d'elle. Elle fait boire une mixture hautement toxique à son pauvre mulâtre de fils d'ailleurs perçu comme un étranger. Lorsque surgit Ousmane, de retour de ses échappées lubriques habituelles, possédant l'énergie du désespoir, elle le gratifie de violents coups de couteaux. Il est pris par un tardif élan de conscience. Pour le narrateur, le délire s'emparant de Mireille est le résultat d'une forme de délire consacré, tranquille et royal: l'intolérance culturelle, voire le racisme de la famille africaine. L'incapacité d'Ousmane à s'affranchir de l'ostracisme nourri par les siens contre sa femme est en soi démentiel. Devant la folie dans laquelle dérape Mireille, c'est plutôt celle de son mari dominateur qui est mise en évidence. Le narrateur est formel:

Elle tremblait, elle hoquetait. En Ousmane un déclic tardif de la raison issu de la peur. Une clarté humaine se frayait enfin une voie dans la 
densité des ombres. Un dégoût nauséeux de lui-même le submergea. Fou à lier, il avait contaminé Mireille. La folie seule expliquait son aveuglement et ses actes. Au- delà de certaines limites, l'engagement est déraison. (246)

On le voit, si la redécouverte des lettres d'amour est psychiquement fatale pour Mireille, son cas est le résultat d'un long processus. Il en est de même chez Sony Labou Tansi.

Les Yeux du volcan apportent une cause assez inattendue à la folie: la frustration sexuelle. Contrainte d'observer une sévère chasteté monastique dans son lit conjugal, Alleando Calero sombre dans la déraison. Et dans ce cas comme dans le précédent, c'est l'homme qui est mis en accusation. Par son attitude, il précipite sa conjointe dans le délire. Mais Sony Labou Tansi présente le cas de son personnage avec une ironie féroce. Car chaque fois que démarrent ses désirs ravageurs, son époux lit résolument et bruyamment la Bible. L'incapacité/le refus de ce dernier d'accomlir son devoir conjugal rend son existence infernale. De frustration en frustration, elle sombre dans le délire et accoplit sa "révolution" à la cuisine:

Alleando Calero coupait très fin ses jupons comme on le fait pour certains légumes. Elle les jetait émincés dans une grande marmite qu'elle avait mise à bouillir sur le feu pricipal de la cuisinière à gaz. Alleando avait déjà coupé ses robes de chambre, ses pagnes, ses blouses, ses bazins, ses madras, ses échappes, ses crimolines. [...] Au moment où l'adjudant arriva, sa femme levait le bras pour jeter dans la marmite ses pierres,ses boucles d'oreilles en or, ses bracelets en or [...]

Alleando ajouta un fagot d'endives et une boîte d'oseille à sa cuisine, puis une pincée de poudre des Césars et trois piments de Nsanga Norda. Ensuite elle versa trois bouteilles entières d'huile d'olive dans la marmite au dessus de laquelle montait une épaisse arborescence de buée appétissante. Elle goûta.

[...] Alleando jeta dans la marmite, sans les émincer, un lot de slips de femmes, de soutiens gorge, et sa provision de tampons périodiques. Elle sépara les rouge à lèvres de leurs étuis avant de les cuisiner. (106-8)

Comme Ousmane, Benoît Goldman est tardivement pris par un désir de se rattrapper. La réaction des deux femmes est identique: violence démentielle contre le persécuteur masculin qui pousse à la rupture psychique. Alleando déchire la lèvre de Goldman qui tente de l'embrasser. On peut le relever avec ces deux cas, le roman africain reconstitue le discours sur la folie. Si la notion de conflit demeure, on observe un élargissement de la communauté à une société civile régie par d'autres paradigmes. Dieu et les ancêtres semblent de plus en plus exclus de la gestion et des enjeux de l'existence. Dans une 
moindre mesure, c'est la famille immédiate mais surtout le dérèglement de l'État qui acculent à la catastrophe mentale. On se situe dès lors dans la perspective de Ronald Laing et de Fanon. Mais la folie comme thème littéraire s'articule-t-elle dans les romans de manière trangressive? Les propos délirants de certains sujets et la déconstruction énonciative peuvent-ils donner lieu à une poétique?

\section{La Folie comme poétique}

Inscrite comme procès d'écriture dans le roman africain moderne, la folie n'est pas uniquement le sens d'une modernité nécessairement négative. Forme singulière des "nouvelles écritures africaines", elle confère simplement au texte fictionnel un statut particulier dans le vaste réseau qui la lie au genre. Shoshana Felman le formule clairement,

Tout roman contient à la fois la tentation de la folie et la négation de celleci, par un système réflexif au sein duquel, d'une façon ou d'une autre, c'est, la folie elle-même qui s'accuse et se dénonce comme telle. Structure schizophrénique, qui se construit pour se détruire, et dont le mode de fonctionnement est celui de sa propre négation. (Felman 126)

Étant donné cette nécessaire intégration de la déraison comme acte narratif et discursif, il est possible, à partir des hypothèses relevées sur l'interprétation de ses causes, d'envisager ses implications sur le fonctionnement textuel.

Il faudrait d'abord admettre que, même si les romanciers et les théoriciens ne se réclament pas de toute théorie psychanalytique, l'effet pathogène du traumatisme psychique peut éclairer la structure narrative des textes faits de nombreuses rétrospections. La psychanalyse classique explique en effet la folie par l'histoire du sujet, par ses antécédents. Même si Oedipe est rigoureusement inopérant en Afrique ainsi que l'indiquent Fanon, M.C. et M. Ortigues ou J. B. Fotso Djemo, nier absolument toute possibilité d'élaboration d'une histoire personnelle comme le fait Sow serait un peu problématique. Car la folie de Zoaétoa dans le texte de Mongo Beti a une histoire, elle est une histoire. Celle-ci concerne un individu empêtré dans les contradictions et les tourments d'un ordre politique pris de panique. C'est pourquoi le titre du roman est en lui même un programme. Dans une telle perspective, la forme narrative essentielle est le retour. Le narrateur annonce dès les premières lignes le caractère rétrospectif de son texte, qui à partir de cet instant, prend une forme linéaire. La cause immédiate de la maladie de Zoaétoa n'est donnée qu'à la fin du roman. Le texte est dès lors une simple explication des origines multiples de l'état clinique du personnage. Dans Le Pacte de sang, Ngandu Nkashama élucide la situation clinique de Mâ Kanzaza et de Vieux Hizambe par le même procédé. C'est ce qui peut expliquer le caractère peu linéaire de ces romans qui tient de l'historicité des personnages. Mais le 
discours du fou a-t-il un impact sur la structure des textes?

Pour l'opinion, le discours du fou est, a priori, indigne d'intérêt, incompréhensible et délirant. Une invalidation et une objectivation faciles l'excluent de la sphère de l'humanité. Un tel discours, produit dans un texte narratif, a des influences sur sa structure syntaxique .Pour l'étude des romans convoqués, on ne considérera, avec Monique Plaza, comme fondamentale dans la folie du discours que le critère de l'inintelligibilité. On relève par exemple une ironie subtile dans le discours du nouveau milliardaire dans_Moha le fou, Moha le sage. Le pouvoir financier constitué en raison se résume dans l'élocution par un ensemble d'incohérences. L'argent rendant fou comme le dit Moha, son interlocuteur est pris dans un délire verbal le contraignant à débiter un tissu d'insanités sans aucun rapport: dieu, l'argent, sa mère, la poésie, l'incurie de l'administration, son passé communiste, une grève, etc. Ce "flot de paroles"fatigue Moha. Il est difficile d'articuler une grammaire cohérente de ce discours (95-100) pourtant tenu par un sujet supposé sain. Ses paroles ne sont pas très différentes de celles que débite le patriarche (66-70) quand une potion magique le pousse dans le délire: de nombreux coq-à-l'âne ponctuent le discours qui font perdre au langage toute fonction référentielle. La même décontextualisation du langage caractérise Magamou dans La Plaie lorsqu'il se retrouve parmi des cadavres à l'hôpital. Ayant perdu conscience, il sombre dans le délire, au plus grand bonheur des agents de l'ordre qui l'avaient d'ailleurs exclu de la sphère de la raison, c'est-à-dire de l'humanité.

Dans Moha le fou, Moha le sage et La Mort faite homme, on observe une oscillation imprévisible et déroutante de la gamme narrative. Les deux romans sont éclatés, voire émiettés. A cause de la rupture du principe de causalité qu'observent Mongo Beti et Malick Fall par exemple, il n'existe pas de rapport entre les différentes séquences textuelles. En l'absence de tout lien entre la consécution et la conséquence, entre l'antécédent et le conséquent, la structure syntaxique des récits est très peu organique. On passe allègrement d'un espace à un autre, d'un narrateur à un autre, d'un morceau narratif à une diversion lyrique. Il existe en outre de nombreux coq-à-l'âne obligeant le lecteur à recoller divers morceaux, et à impliquer dans les romans suffisamment son "encyclopédie personnelle" (Eco). La superposition, voire la juxtaposition des unités sémantiques embrouille la compréhension immédiate. Une telle transgression dans les langages éloigne des théories de Michael Rifaterre sur "la production du texte", car la compréhension du nouvel univers syntaxique et logique oblige à l'élaboration de nouvelles grilles des mécanismes d'engendrement textuel. Du point de vue strictement narratif, on ne sait pas toujours exactement "qui parle " et les points de vue oscillent interminablement. Un tel choix esthétique inflige au lecteur une bonne part de la torture décrite dans les romans. D'où la nécessité de constituer de nouveaux paradigmes face à la déconstruction et au dysfonctionnement tant de l'énoncé que de l'énonciation.

Le roman africain à l'épreuve de la folie produit donc un langage autre, celui de la rupture avec soi et avec le monde objectif. Elle est systématisée par l'abandon des principes logiques et syntaxiques qui transforment la déraison en "dérision esthétique". Mais au bout de cette structure 
schizophrénique se trouve le délire, l'inintelligible. C'est lui qui marque l'étape finale de la souffrance de Mianza dans La Mort faite homme. Il est consacré par une syntaxe hasardeuse qui segmente anarchiquement les termes et les phrases, de sorte qu'on ne puisse rien en tirer de sensé. C'est ce qui y consacre la "folie textuelle" totale, de loin plus expressive que les incohérences du patriarche et du nouveau milliardaire de Ben Jelloun:

\author{
Mon amour Mianza. Amour. Mon enfant. Ma fille. D'amour. Ta main. Par \\ là Oh Mon Ami. Ami de juin. Juin de sang de feu. ..mon. Les Immondes \\ Monde. Immondices. [...] Car carca [...] Je. Te. T'ai. Me. A. Mi... les yeux. \\ Tes yeux pleurent. Mes yeux larmes. Ta main. A. Mie. Pleu. Rant. Mon \\ Ge... Mon. A. Mi. Mia.Mi. Mian. A. Mour. Fi...Ille. Fem. Me...Ta. Ta. Ta. \\ Ta. (256).
}

On le voit, l'inintelligible, plus que le brouillage de l'identité et de la perspective énonciative attestent mieux l'inévitable incorporation de la folie à la littérature. L'articulation d'une poétique de la folie est donc possible. La subversion des genres, la transgression des codes sont, ainsi que l'indiquent Felman, non pas "origine de l'écriture, cause du sens, mais effet de discours..." (Felman 188, italiques dans le texte). Mais cet effet du discours est lui-même lieu d'un discours, objet d'un discours portant sur deux questions qui lui sont intomenent liées: la vérité et la liberté.

\title{
Folie, Vérité et Liberté
}

La folie comme thématique littéraire en Afrique couvre non seulement les conflits interpersonnels, mais aussi les discours. Celui du fou est transgressif à plus d'un titre. L'enjeu des rapports existentiels de Sarzan, Zoaétoa, Mireille et d'Alleando est essentiellement un rapport à la vérité: la renverser, lui résister ou la dominer. Et depuis le "coup de force" cartésien dont parle Foucault, une ségrégation emmure le fou réel ou imaginé dans la catégorie de la non pensée. Or il subvertit les convictions collectives par un discours iconoclaste. Paria ayant perdu toute inhibition psychomotrice, il a la facilité de dévoiler candidement ou avec conviction ce qu'on voudrait bien taire. À ce titre, le fou est un prophète, l'incarnation d'un évangile et mérite d'être protégé contre la communauté. Il porte la vérité du monde, dans toute sa verdeur et sa nudité. Et il est significatif que les romans convoqués ici couplent la nudité des fous à la poétique. L'exhibition gracieuse des anatomies y permet d'infliger à l'homme sa vérité originelle.

D'autre part, on se rend compte que ce sont les "fous"paradoxalement mis au banc de la société qui perçoivent avec une rare lucidité les délires et les errements sereins dans lesquels se prélasse "la majorité déviante". Ironie fondamentale. Ainsi par exemple, dans Les Yeux du volcan, c'est Rouviera Mendèsdo di Sandoval, épileptique malade mental 
connu de tous, qui tourne la foule en dérision en lui révélant sa couardise et sa capacité à se se construire des rêves. Exploitant les fantasmes de libération des populations, il se déguise en Igancio Banda, libérateur mythique attendu par une masse rassemblée sur la place publique pour la circonstance. Il enlève ses masques et crie à la foule la vérité de sa couardise. Magamou quant à lui est scandalisé par l'incroyable servitude de la femme, ainsi que par les mystifications post-coloniales. Son malheur supprême vient justement de la sévérité des critiques qu'il formule contre la société et les institutions. Celles-ci font vite de médicaliser sa déviance et de psychiatriser une simple blessure sommatique. Comment parler de folie dans ces cas? La question révèle toute son ambivalence et les textes insinuent que la déraison n'est pas toujours là où on la soupçonne. Gianni Scalia:

Le problème de la maladie et de la folie est le problème du rapport avec la "vérité" en tant que sens, mode d'apparaître, du lieu du langage de la vérité à qui il faut donner la parole et dont la parole doit être déchiffrée.(Scalia 151, italique dans le texte)

Le roman de Ben Jelloum est à ce sujet révélateur. Moha le fou, Moha le sage est en soi une ironie sévère permettant de reconsidérer la dialectique raison-folie. Foucault l'a démontré, la folie est aussi ségrégation consécutive à la trangression du discours. Thomas Szasz va plus loin en démontrant que les instances sociales inventent le mythe de la maladie mentale pour voiler ou étouffer les contradictions qui les menacent. Et si le dans le texte de Ben Jelloum, comme ailleurs, le pouvoir se constitue en raison, la vérité est par contre rendue par les marginaux. Roland Jaccard a à ce sujet une formulation heureuse: "le fou nous rappelle que quelque chose ne va dans la rationalité dominante. Que derrière la façade se cache une autre réalité; implicitement, il conteste nos certitudes et dit des choses inopportunes et scandaleuses que nous ne voulons pas écouter" (Jaccard 7).

En fait, Moha "le fou" est plutôt un visionnaire inquiet de la catastrophe vers laquelle se dirige paisiblement une société rongée par le pouvoir et l'argent. Ainsi qu'il le dit lui même, il n'est qu'un messager. Et qu'il ait de si nombreux sympathisants est un signe: des enfants le suivent pour écouter sa poésie; des foules nombreuses se massent sur sa tombe. Cela montre que sa "folie" comporte quelque chose de profond, de séductif et d'hallucinogène. La "raison" collective et sociale est dans ce cas un leurre. Il le dit d'ailleurs: "Je suis fou. Comme je suis seul à être fou, c'est que je dois avoir raison. L'unanimité m'inquiète." (44). Le monologue de la raison sur la folie, la prétention à la vérité de la part de divers sujets trouve dans le roman de Ben Jelloun des formes pertinentes et troublantes. Et est significative, à ce titre, la relation de Moha au nouveau milliardaire. Ce dernier lui déclare en effet:

Quand je te vois, je me sens atteint de lucidité. C'est le pouvoir. Il me rend fou. Mais pas le genre de ta folie. Elle est belle et saine. La tienne.[...] Je 
suis seul [...] Le pouvoir, ça rend fou. (124)

Cette confession révèle davantage qu'un homme inquiet: un prisonnier. L'homme est aliéné par sa propre fortune. Paradoxalement, Moha, qui a fait voeu et option de pauvreté matérielle, est libre, absolument. C'est cette liberté qui lui permet de dire la vérité du monde, d'autant que les autres membres de la société, pour des restrictions de toutes sortes, sont incapables d'articuler cette vérité et même de la percevoir. Alors Moha se révèle le porte parole auquel on s'identifie silencieusement. Et c'est la "folie" supposée de Moha qui garantit sa liberté car la vérité n'est tolérée que tant qu'elle émane d'un sujet dont le discours est invalidé du fait du corset dans lequel on l'enferme. Mais la relation de la folie à la liberté peut aussi tenir d'une ruse.

En effet, le roman africain moderne ne fait pas uniquement de la folie une question philosophique, clinique ou une dissidence délibérée. Il opère également un truquage positif de la question de la maladie mentale. L'argument ici est la récupération du préjugé défavorable et de la disqualification systématique dont est victime le fou. Des personnages font ainsi, contre mauvaise fortune bon coeur. La subversion de la maladie mentale dont parle Szasz n'est plus le fait des pouvoirs totalitaires. Elle est plus qu'une ruse pouvant s'avérer meurtrière pour l'oppresseur: une invention idéologique servant à la reconquête de la liberté. Le fou étant généralement considéré comme inoffensif, on capitalise sur cette présomption. Rousseau l'indiquait déjà, "se rendre fou pour devenir sain" peut s'imposer comme nécessité existentielle. Scalia décrit le processus:

À la limite, la raison se "renverse" radicalement, se pense comme limite de la raison, exprime la fureur et l'horeur de la raison défaite par la nature ou société "méchante", féroce, atroce. C'est l'horreur décrite par Sade, c'est l'accusation et la condamnation des fous. On se fait fou pour s'opposer à la société devenue rationnellement folle...(Scalia 149. Italiques dans le texte)

Ainsi par exemple, Magamou, déclaré fou et incarcéré tout à fait arbitrairement, est-il contraint d'assumer le personnage du fou que la société lui a collé pour se libérer. Les recommandations du gardien sont formelles, seule une mise en scène de la folie peut le sortir du cabanon:

Bon. Un conseil. Ne te fais pas d'illusion. Si tu acceptes de passer pour un fou, tout le monde va dire: "amen". "Voilà un fou raisonnable"[...] si tu prétends que tu jouis de toutes tes facultés, le toubib t'apprendra que tu as réagi exactement comme un fou. Si tu gardes le silence, tu seras considéré comme un fou inoffensif. Si tu t'emballes, si tu tentes de démontrer quoi que ce soit, tu passeras pour un fou furieux, mûr pour les fers. (89-90) 
Magamou s'exécute. Il grimace l'aliénation, distrait les gardiens et s'évade. Le même travestissement de la maladie mentale s'opère chez Sony Labou tansi.

Dans L'Anté-Peuple, la folie est d'abord une stratégie de survie et de sécurité. Les "fous" qui parcourent la ville, tout nus, sont en fait des personnes saines contraintes de s'aliéner. La cause: la cité est infestée de miliciens qui demandent les "papiers" à chaque centimètre. Dans cet État policier vivant dans la psychose du putch, seuls sont épargnés les fous. Alors pour reconquérir la liberté de mouvement, il faut affecter la folie et offrir sa nudité comme signe distinctif. Mais il y existe un meilleur usage de la maladie.

En effet, sur instruction de la hiérarchie du maquis, Dadou doit se travestir en devenant "fou" pour accomplir sa mission historique. Tous les déments étant présumés inoffensifs, les maquisards doivent en outre capitaliser sur les bonnes dispositions du Premier à l'égard de ces derniers. Homme d'État cherchant à se faire bonne conscience, contrairement à certaines habitudes, Le Premier traite humainement les fous et leur donne même de l'aumône. Comme Magamou, Dadou grimace patiemment la folie, guette le politicien jusqu'à l'assassiner en pleine église. On peut dès lors se poser une question: l'intégration des fous, l'humanisation des rapports avec eux peut-elle être dangereuse? Production sociale, la folie comme ségrégation est-elle aussi nécessité? En tout cas, le roman africain indique ici que la ségrégation et la marginalisation du dément peut servir des causes heureuses. Il est le lieu de déploiement d'une dynamique multiple de la démence qu'il relativise et aborde dans toutes ses coutures. Non seulement il élabore élabore une poétique et même un savoir comme l'indique Mouralis, mais l'interprétabilité des corrélats littéraires de l'aliénation mentale renvoie en parmanence à des hypothèses théoriques.

\section{Conclusion et conséquences théoriques}

Pourquoi continuer à lire, trente cinq ans après Fanon, la condition africaine sous le prisme de la déraison? Mongo Beti donne la réponse dès les pages liminaires de L'Histoire $d u$ fou: 'I'indépendance, c'est à elle qu'il faut toujours revenir, comme à la source de nos malheurs[...] l'indépendance ressembla fort à la mort: impossible d'imaginer ce qu'il y aurait après, personne n'en étant jamais revenu à sa connaissance"(13). Ce qu'elle a apporté au Nègre, $c^{\prime}$ est un cortège de malheurs et la mort psychique, plus dangereuse que la mort physique. Même l'esprit le plus flegmatique ne saurait résister à cette vague de folie mettant en péril l'équilibre mental. Il est plus explicite encore:

Il est vrai aussi que la spirale de fureur et de démence, dont l'indépendance fut le signal, créa un climat inhumain auquel bien peu d'esprits purent résister. C'est ce dont témoigne ce peuple de fous qui errent comme des spectres dans les rues de nos villes et qui ont une histoire tragique [ ...] les foules on été précipitées dans le cauchemar et s'y débattent encore. (78) 
On croirait lire Fanon! En tout cas, ces réflexions renforcent l'hypothèse énoncée au départ. Il semble donc important de réordonnancer le discours théorique sur la démence en Afrique. Car si pour Sow, elle est consécutive au surgissemnt d'un chaos préculturel, l'adoption de ses paradigmes épistémologiques éluciderait mal les cas abordés dans cette étude. Davantage, il semble exclure l'ordre politique de l'ordre culturel. Or il est évident que l'avènement d'une société civile chaotique et de nouveaux modes de gestion instaurent un renversement essentiel aussi bien dans les relations inter-personnelles que dans les attitudes au monde. Même s'il ne se démarque pas de façon décisive des hypothèses de Sow portant uniquement sur le "milieu tradionnel", Fotso Djemo envisage la nécessité d'inclure le paramètre politique dans l'élucidation de la maladie mentale. À la suite de Fanon, et tout en disqualifiant une certaine psychanalyse, il incorpore le paradigme idéologique dans sa grille en énonçant " une prise de position sans équivoque de l'économique, du politique, du religieux, du scolaire, etc [...] en faveur de la lutte de libération des peuples [...]c'est-à-dire l'inscription des valeurs de libération dans ces différents secteurs [...]" (Fotso Djemo 423).

Les textes abordés ici l'indiquent clairement, la folie, "que l'on peut interpréter comme une métaphore du dysfonctionnement des sociétés africaines" (Mouralis 13) est de plus en plus une question idéologique et politique. L'inauthenticité des rapports sociaux accule les sujets à la rupture psychique. Ceux-ci peuvent également se voir précipités dans le délire par la violence d'un fait et d'un instant. Davantage: le roman africain révèle plutôt une entrée troublante dans la modernité. L'avènement de la société civile subvertit de façon décisive les fondements du politique, car pour survivre, le citoyen doit absolument se protéger contre l'État. La question de la folie devient donc emminement politique en Afrique. Elle est consécutive au dysfonctionnement et au chaos institutionnels. Alors qu'en Occident, la démence est liée à l'expansion anarchique du capital, en Afrique, elle est liée au désordre généré et entretenu par le politique.

Par ailleurs, les romans considérés autorisent une lecture psychanalytique. Peut-être pas comme cure, mais certainement comme interprétation, ainsi que le soulignait Fanon. Jacques Lacan l'indiquait, il existe un "au-delà du sujet" à considérer. Et le sujet africain d'aujourd'hui n'est plus nécessairement prisonnier de son lignage ou de son groupe ethnique. Il se constitue lui même en tant qu'histoire, individualité se débattant dans un contexte social investi par de grandes paniques. La crise par la folie se traduisant dans le texte littéraire par une transgression des langages et des codes ne saurait donc être une pure incidence. Les "nouvelles écritures africaines" sont dès lors des manifestations d'un combat pour une survie improbable chez des peuples menacés jusqu'à leur santé mentale. La poétique de la folie acquiert donc une fonction transitive. Herbert Marcuse l'écrit, "La forme esthétique continuera à se modifier au fur et à mesure que la pratique politique réussira, ou échouera, à bâtir une société meilleure. Dans le meilleur des cas, on peut envisager un univers commun à l'art et à la réalité [...]" (Marcuse152)

On le voit, la folie en Afrique et l'interprétabilité de son fontionnement dans les textes 
fictionnels ramènent sans cesse à des cadres institutionnels et idéologiques. Fanon l'a

- formulé depuis longtemps et des études autorisées l'ont suivi dans ce sens, la folie est inséparable de la gestion de la cité, laquelle conditionne le psychisme. La vanité de l'exercice de la psychiatrie dans le cadre colonial se reproduit après les indépendances, car l'impression permanente d'apocalyse demeure à travers la prolifération d'institutions carcérales comme dans les textes de Ngandu Nkashama. Mongo Beti est sans équivoque sur la question, l'indépendance a été une épouvante juridique et mentale. Les textes de Mariama Bâ et de Labou Tansi indiquent davantage: la nécessité d'humaniser les relations entre partenaires, ainsi que l'instauration d'une société de droit. Étant donné la fureur décimant l'esprit humain, la panique s'emparant du psychisme et "l'ensauvagement" de l'humanité, l'écrivain congolais en arrive à conclure simplement que Descartes s'est rigoureusement trompé sur l'universalité du bon sens. Dans un tel contexte, écrire aujourd'hui, en parlant de l'Afrique, comme le fait Fotso Djemo, qu'il n'est "Pas question de trouver dans le politique la solution mirarcle aux problèmes de santé mentale ni de vouloir refaire les sciences théoriques et pratiques à partir d'une base idéologique à priori " (419) peut sembler quelque peut ahurissant en soi. Face à "un monde beaucoup plus terrifiant que le monde colonialiste" (Les Damnés...30), le sociodiagnostic que pose Fanon se reproduit dans son intégralité et cette étude adhère entièrement à cette optique: "Il n'y aura d'authentique désaliénation que dans la mesure où les choses, au sens le plus matérialiste, auront repris leur place" (Peaux noires...9). Tel est le discours que semblent à la fin suggérer les textes littéraires africains contemporains sur la question de la déraison.

\section{Notes}

${ }^{1}$ Cet article est la version d'une communication présentée lors de l'atelier "Identités francophones" du dernier congrès annuel de l'Association Canadienne de Littérature Comparée à Ottawa le 30 mai 1998.

2 Esthétique et folie dans l'oeuvre romanesque de Pius Ngandu Nkasahama. ParisMontréal: L'Harmattan, 1998. 


\section{Romans}

\section{Références}

Bâ, Mariama. Un Chant écarlate. Dakar: NEA, 1981.

Ben Jelloum, Tajar. Moha le fou, Moha le sage. Paris: Seuil, Coll. Points, 1978.

Beti, Mongo. L'Histoire du fou. Paris: Julliard, 1994.

Conchon, Georges. L'État sauvage,. Paris: Albin Michel, 1964.

Diop, Birago. "Sarzan”, Les Contes d'Amadou Koumba. Paris: Présence Africaine, 1969. 173-187.

Fall, Malick. La plaie .Paris: Albin Michel, 1967.

Grainville, Patrick. Les Flamboyants. Paris: Seuil, 1976.

Labou Tansi, Sony. L'Anté-Peuple. Paris: Seuil, 1983.

. Les Yeux du volcan. Paris: Seuil, 1988.

Ngandu Nkashama, Pius. Le Pacte de sang. Paris: L'Harmattan, Coll. Encres Noires 25, 1984.

- La Mort faite homme. Paris:L'Harmattan, Coll. Encres Noires 38, 1986.

\section{Études sur la roman africain}

Dabla, Séwanou. Nouvelles Écritures africaines. Romanciers de la deuxième génération. Paris: L'Harmattan, 1986.

Kom, Ambroise. "Folie et révolution: Sahel! Sanglante sécheresse de Mandé Alpha Diara et Les Chauves Souris de Bernard Nanga". Littératures africaines. Paris: Silex, 1988: 6574; "Entre traîtres et fous: les deux romans de Bernard Nanga". Romanisches Jahrbuch, Band 41: 139-147.

Ngandu Nkashama, Pius. Écritures et discours littéraires. Études du roman africain. Paris: L'Harmattan, 1989.

Tcheuyap, Alexie. Esthétique et folie dans l'oeuvre romanesque de Pius Ngandu 
Nkashama. Paris: L'Harmattan, 1998.

Utudjian, Eliane Saint-André. (1980), "Le thème de la folie dans la littérature africaine contemporaine". Présence Africaine 115. Paris: Présence Africaine: 118-147.

\section{Textes théoriques}

Basaglia, Franco et Basaglia-Ongaro, Franca. La Majorité déviante. L'idéologie du contrôle social total. Paris: UGE 10/18, 1976.

Eco, Umberto. Lector in fabula. Le rôle du lecteur. Paris: Grasset et Fasquelle, 1985.

Felman, Shoshana. La Folie et la chose littéraire. Paris: Seuil, 1978.

Fanon, Frantz. Peaux noires, Masques blancs. Paris:Seuil, Coll. Points, 1959.

Les Damnés de la terre. Paris: Maspéro, 1961.

Fotso Djemo, Jean Baptiste. Le Regard de l'autre. Médecine traditionnelle africaine. Paris: ACCT-Silex, 1982.

Foucault, Michel. Histoire de la folie à l'âge classique. Paris: Gallimard, Coll. Tel., 1972.

Jaccard, Rolland. La Folie. Paris: PUF, Que sais-je?, 3ème éd., 1984.

Marcuse, Herbert. Contre Révolution et révolte. Paris: Seuil, Coll. Combats, 1973.

Mouralis, Bernard. L'Europe, l'Afrique et la folie. Paris: Présence Africaine, 1993.

Ortigues, M.C et E. Oedipe africain. Paris: Plon, 1973.

Plaza, Monique. Écriture et folie. Paris: PUF, 1986.

Rifaterre, Michael. La Production du texte. Paris: Seuil, 1979.

Sémiotique de la poésie. Paris: Seuil, 1983.

Scalia, Gianni. "La raison de la folie". La Majorité déviante. L'idéologie du contrôle social total. Op. cit: $143-188$.

Sow, Tbrahima. Psychiatrie dynamique africaine. Paris: Payot, 1977.

Structures anthropologiques de la folie en Afrique noire. Paris: Payot, 1978.

Szasz, Thomas. Le Mythe de la maladie mentale. Paris: Payot, 1986 